\title{
Pharmacokinetic and Pharmacodynamic Study of Terazosin in Healthy Chinese Volunteers: Significant Hysteresis Phenomenon
}

\section{Guifen Qiang, Man Yang, Yanan Zhang, Man Liu, Dan Zhang, Guocai Wang, Jing Han, Xue Xiao, Zhenlong Wang and Huichen Liu*}

Department of Clinical Pharmacology, Aerospace Center Hospital, Beijing, China

\begin{abstract}
Aim: The present study investigated the correlation between plasma concentration and antihypertensive effect of terazosin in healthy Chinese volunteers.

Methods: A randomized, single-dose pharmacokinetic study of $2 \mathrm{mg}$ terazosin hydrochloride tablet was conducted in healthy Chinese male subjects. Supine blood pressure and heart rate were measured before and after dosing, using cardiogram monitor. Serial blood samples were collected and plasma concentrations of terazosin were quantified by high performance liquid chromatography (HPLC) with a fluorescence detector. Non-compartmental pharmacokinetic analysis was applied to plasma concentrations.

Results: Plasma concentration of terazosin reached to peak concentration at $0.89 \pm 0.62$ hours $\left(T_{\text {max }}\right.$ ), and then decreased with half-life value of $10.21 \pm 1.41$ hours. Systolic blood pressure (SBP) and diastolic blood pressure (DBP) decreased from 0.25 hours till 24 hours after dosing. The maximum decrease of $8.5 \pm 5.3 \mathrm{mmHg}(7.78 \%)$ in SBP $(P<0.05)$ and $12.9 \pm 6.0 \mathrm{mmHg}(19.82 \%)$ in DBP $(P<0.001)$ occurred at 8 hours after dosing with a lagging time of 7 hours than $T_{\text {max }}$, different from 1.2-1.8 hours of lagging time in healthy Spanish subjects. The heart rate increased from 0.25 hours till 24 hours with the maximum increase of $14.3 \pm 7.8$ beats $/ \mathrm{min}(23.85 \%)$ at 6 hours after dosing.

Conclusion: The findings suggested that terazosin had a significant hysteresis phenomenon of antihypertensive effect behind plasma concentration of terazosin in healthy Chinese subjects, as was different from other racial subjects.
\end{abstract}

Keywords: Pharmacokinetic; Pharmacodynamic; Terazosin; HPLC

\section{Introduction}

Terazosin, a post-synaptic $\alpha_{1}$-adrenoceptor antagonist, is used to treat the essential hypertension and the symptom of benign prostatic hyperplasia (BPH) [1,2]. Compared with doxazosin, alfuzosin and tamsulosin, terazosin is the drug with a favorable effect in four areas of interest, including BPH symptoms, blood pressure, platelet aggregation, and endothelial functions in the patients with $\mathrm{BPH}$ [3]. In addition, terazosin was proved to be effective and safe in the treatment of lower urinary tract symptoms (LUTS) in male or female patients [4,5].

As an antihypertensive drug, terazosin monotherapy or in combination with other antihypertensive agents significantly reduces the blood pressure, especially diastolic blood pressure in the patients with mild to moderate hypertension $[1,6]$. The antihypertensive effect and the dose of terazosin showed a strong dose-response relationship in the dose of 1 to $10 \mathrm{mg}$, with a maximum response plateau above $10 \mathrm{mg}$. The maximum antihypertensive response $\left(\mathrm{E}_{\max }\right)$ to terazosin was $10.7 \mathrm{mmHg}$ for SBP and $8.0 \mathrm{mmHg}$ for DBP in the patients with mild to moderate essential hypertension [1]. Besides the hypertensive patients, terazosin can also decrease the blood pressure in healthy subjects. A single $0.5 \mathrm{mg}$ terazosin can decrease the supine and $50^{\circ}$ tilt DBP, while higher doses were required to decrease SBP in healthy Japanese volunteers [7]. It is worth noting that syncope and orthostatic hypotension, as serious adverse events, are prone to occur after the first dose of terazosin, namely "first-dose" effect [8].

The pharmacokinetic study indicates that plasma concentration of terazosin increases proportionately with oral doses up to $5 \mathrm{mg}$. After oral administration, terazosin is rapidly absorbed to reach the maximum concentration at 1-2 hours. Approximately $90 \%$ to $94 \%$ of terazosin is bound to plasma protein, with the volume of distribution estimated to be 25 to 30 liter [9]. The mean beta-phase half-life is approximately 12 hours. The prolonged half-life of terazosin allows once-daily dosing, which may facilitate the patients compliance with drug therapy for hypertension [9]. The elimination half-life of terazosin is not significantly altered in the patients with hypertension and renal insufficiency $[8,10]$. However, the pharmacokinetic parameters of terazosin were influenced by age. The older healthy subjects have the higher mean and peak plasma concentrations of terazosin and the longer elimination half-life [11]. Thus, the suitable dosage of terazosin should be taken for the elderly hypertensive patients.

Single-dose study in healthy subjects is particularly valuable for investigating the concentration-effect relationship. The blood samples can be taken repeatedly more easily than in the patients. The pharmacokinetic or bioequivalence studies of terazosin have been conducted in healthy Chinese subjects to investigate the pharmacokinetic property of terazosin. However, the correlation between the plasma concentration of terazosin and the antihypertensive effect has not been fully elucidated. In the present study, we measured the blood pressure to assess the antihypertensive activity and determined the plasma concentration of terazosin following

*Corresponding author: Huichen Liu, Department of Clinical Pharmacology, Aerospace Center Hospital, 15 Yuquan Road, Haidian District, Beijing 100049 China, Tel: (86)10-59971772; Fax: (86)10-59971773; E-mail: huichenliu@163.com

Received September 18, 2011; Accepted November 01, 2011; Published November 03, 2011

Citation: Qiang G, Yang M, Zhang Y, Liu M, Zhang D, et al. (2011) Pharmacokinetic and Pharmacodynamic Study of Terazosin in Healthy Chinese Volunteers: Significant Hysteresis Phenomenon. J Bioequiv Availab 3: 228-232. doi:10.4172/ jbb.1000091

Copyright: (C) 2011 Qiang G, et al. This is an open-access article distributed under the terms of the Creative Commons Attribution License, which permits unrestricted use, distribution, and reproduction in any medium, provided the original author and source are credited. 
the administration of $2 \mathrm{mg}$ terazosin hydrochloride tablet in healthy Chinese male subjects, in order to investigate the pharmacokinetic and pharmacodynamic response of terazosin.

\section{Material and Methods}

\section{Subjects}

Eligible subjects were healthy men aged 18 to 40 years. They were required to have values within the following ranges during screening: a body mass index (BMI) of 18 to $24 \mathrm{~kg} / \mathrm{m}^{2}$, and no clinically significant abnormal findings on the physical examination, medical history, clinical laboratory results (including test of blood routine, urinalysis, hepatic and renal function), or 12-lead electrocardiogram (ECG) examination. Key exclusion criteria included the history of orthostatic hypotension, history of long-time smoking, the presence of disorders that had the potential to affect the absorption, metabolism, or elimination of study drugs or that could constitute a risk to the subject when taking the study drug. No prescription or over-the-counter medications was permitted within 14 days and no consumption of alcohol within 24 hours prior to administration of study drug. Subjects were not allowed to consume alcohol or tobacco, or take any other medication during the study.

\section{Study design}

Twenty-two healthy male Chinese volunteers participated in the randomized, single-dose pharmacokinetic study under fasting condition. After an overnight fast, a single oral dose of terazosin hydrochloride tablet (HYTRIN ${ }^{\oplus}$, Shanghai Abbott Pharmaceutical Co. Ltd, China), containing $2 \mathrm{mg}$ terazosin, was administered with $200 \mathrm{~mL}$ luke water. The subjects remained in a comfortable recumbent position for up to 8 hours after dosing and remained under medical surveillance throughout the study period. Before they were allowed to ambulate, they sat up with a dependent position for one minute prior to standing up. Vital signs, including supine blood pressure and heart rate, body temperature and respiration, were measured before dosing and at 1 , 3, 24 and 48 hours after dosing. The subjects remained in the clinical research unit until 48 hours after dosing.

The study protocol was approved by the Ethics Committee of Aerospace Center Hospital (Beijing, China). All volunteers gave written informed consent after the aims and risks of the study were fully explained. The study was conducted in accordance with the applicable laws and regulations, with good clinical practice (GCP), and with the ethical principles originating in the Declaration of Helsinki.

\section{Sample collection}

Serial blood samples ( $4 \mathrm{~mL} / \mathrm{sample}$ ) for pharmacokinetic analysis were collected by an indwelling catheter placed on the forearm, into the heparinized tubes before dosing and at $0.1,0.25,0.5,0.75,1,1.5$, $2,3,4,6,8,12,24,36$ and 48 hours after dosing. The blood samples were separated by centrifugation at $3000 \mathrm{~g}$ for $10 \mathrm{~min}$ at $4^{\circ} \mathrm{C}$, then the plasma samples were transferred to polystyrene microcentrifuge tube and stored immediately at $-70^{\circ} \mathrm{C}$ until analysis.

\section{Pharmacodynamic evaluation}

Supine blood pressure and heart rate were measured before dosing and at $0.25,0.5,0.75,1,1.5,2,3,4,6,8,12,24$ and 48 hours before sampling, using non-invasive oscillometric method by cardiogram monitor (PM-9000 Express, Shenzhen Mindray Co., Ltd). The investigators responsible for measuring the blood pressure participated in the uniform training.

\section{HPLC analysis of terazosin}

Terazosin (99.3\%) hydrochloride and prazosin hydrochloride (100\%), internal standard (I.S.), were purchased from National Institute for the Control of Pharmaceutical and Biological Products (Beijing, China). All solvents and other chemicals were HPLC or analytical grade.

Plasma concentrations of terazosin were quantified by a slight modification of the reported HPLC method [12]. In brief, a $200 \mu \mathrm{L}$ aliquot of plasma sample was added with a $100 \mu \mathrm{L}$ aliquot of $45.0 \mathrm{ng} /$ mL I.S. solution, a $50 \mu \mathrm{L}$ aliquot of $1 \mathrm{~N} \mathrm{NaOH}$, and a $3 \mathrm{~mL}$ aliquot of aether-dichloromethane $(3: 2 ; \mathrm{v} / \mathrm{v})$. After vortex for $10 \mathrm{~min}$ and centrifugation at $3500 \mathrm{~g}$ for $5 \mathrm{~min}$, the organic layer was collected and dried under a gentle stream of nitrogen gas at room temperature. A $150 \mu \mathrm{L}$ aliquot of methanol-water $(1: 1 ; \mathrm{v} / \mathrm{v})$ was added to reconstitute the residue and a $50 \mu \mathrm{L}$ aliquot was directly injected onto an Apollo C18 column ( $150 \mathrm{~mm} \times 4.6 \mathrm{~mm}$ i.d., $5 \mu \mathrm{m}$, Alltech, USA) for HPLC analysis. Terazosin and I.S. were separated on the column using 50 $\mathrm{mM} \mathrm{K}_{2} \mathrm{HPO} 4$ : acetonitrile $(78: 22 ; \mathrm{v} / \mathrm{v})$ as mobile phase at a flow rate of $1.1 \mathrm{~mL} / \mathrm{min}$. The column effluent was monitored using a fluorescence detector set at an excitation wavelength of $238 \mathrm{~nm}$ and an emission wavelength of $370 \mathrm{~nm}$. The retention time of terazosin and I.S. was approximately 4.2 and $7.4 \mathrm{~min}$, respectively. The LLOQ was $0.5 \mathrm{ng} / \mathrm{mL}$ and the linear relationship was obtained in the terazosin concentration range from 0.5 to $125 \mathrm{ng} / \mathrm{mL}$, with the correlation coefficient 0.999 . The recovery of terazosin from human plasma was in the range of 86.9 to $89.5 \%$. For the quality control (QC) samples, intra- and inter-day precision as relative standard deviation (RSD) was $<6.7 \%$ and accuracy as relative error (RE) was within $\pm 13.0 \%$.

\section{Pharmacokinetic analysis}

Pharmacokinetic parameters were calculated using noncompartmental method derived in DAS 2.1.1 (Mathematical Pharmacology Professional Committee, Chinese Pharmacological Society). The maximum plasma concentration $\left(\mathrm{C}_{\max }\right)$ and the time to reach $\mathrm{C}_{\max }\left(\mathrm{T}_{\max }\right)$ were determined from the observed plasma concentrations of terazosin. The area under the plasma concentrationtime curve $\left(\mathrm{AUC}_{0-\mathrm{t}}\right)$ was obtained by the linear trapezoidal rule up to the last sampling point with detectable levels. The $\mathrm{AUC}_{0-\mathrm{t}}$ was extrapolated to infinity $\left(\mathrm{AUC}_{0-\infty}\right)$ by adding the quotient of last measurable plasma concentration $\left(\mathrm{C}_{\mathrm{t}}\right)$ and the terminal elimination rate constant (Kel). Kel was estimated by a non-linear least square regression analysis of the individual concentrations observed as a function of time during the elimination phase. The corresponding elimination half-life $\left(t_{1 / 2}\right)$ was calculated as $0.693 / \mathrm{Kel}$. The oral apparent clearance $(\mathrm{CL} / \mathrm{F})$ and volume of distribution $(\mathrm{V} / \mathrm{F})$ were derived from the ratio dose/ $\mathrm{AUC}_{0-\infty}$ and dose/( $\left.\mathrm{AUC}_{0-\infty} \cdot \mathrm{K}_{\mathrm{e}}\right)$, respectively.

\section{Safety}

Adverse events (both observed and spontaneously reported) and their resolutions were recorded throughout the study. An adverse event was defined as any untoward medical occurrence associated with the use of a drug, whether or not considered related to the study drug. Safety evaluations, including physical examination, measurement of vital signs, examination of 12-lead ECG and laboratory test, were performed before discharge from the clinical research unit. Clinically significant findings would be rechecked at follow-up visit until they became normal. 
Citation: Qiang G, Yang M, Zhang Y, Liu M, Zhang D, et al. (2011) Pharmacokinetic and Pharmacodynamic Study of Terazosin in Healthy Chinese Volunteers: Significant Hysteresis Phenomenon. J Bioequiv Availab 3: 228-232. doi:10.4172/jbb.1000091

\section{Statistical analysis}

All parameters were expressed as mean \pm standard deviation (SD). Statistical analysis was performed using SPSS 13.0 software. The statistical difference was evaluated using one-way analysis of variance (ANOVA) and $P$ value less than 0.05 was considered statistically significant.

\section{Results}

\section{Subjects}

The study enrolled 22 healthy subjects. The mean (SD) age of healthy subjects was 25(3) years, their mean body weight was 64.6 (5.6) $\mathrm{kg}$, and their BMI was $22(2) \mathrm{kg} / \mathrm{m}^{2}$. One subject was withdrawn due to having a fever and using the antipyretic drug during the study period. Thus, 21 subjects completed the study.

\section{Pharmacodynamic evaluation}

The mean baseline of supine SBP, DBP and heart rate was $109.3 \pm 9.4 \mathrm{mmHg}, 65.1 \pm 5.5 \mathrm{mmHg}$ and heart rate $60.1 \pm 4.1$ beats $/ \mathrm{min}$, respectively.

After oral administration of $2 \mathrm{mg}$ terazosin, SBP and DBP decreased from 0.25 hours till 24 hours. Compared with the baseline blood pressure, SBP showed a significant reduction $(P<0.05)$ only at 8 hours, whereas DBP had a significant reduction $(P<0.05)$ at the time intervals of 1-12 hours after dosing. Terazosin caused the maximum decreases of $8.5 \pm 5.3 \mathrm{mmHg}(7.78 \%)$ in SBP $(P<0.05)$ and $12.9 \pm 6.0 \mathrm{mmHg}(19.82 \%)$ in DBP $(P<0.001)$ at 8 hours after dosing. The individual maximum reduction was $22 \mathrm{mmHg}$ in $\mathrm{SBP}$ and $23 \mathrm{mmHg}$ in $\mathrm{DBP}$, respectively (Figure 1).

After dosing, heart rate increased from 0.25 hours till 24 hours, with a significant increase $(P<0.05)$ at the time intervals of $0.75-12$ hours. Terazosin caused the maximum increase of $14.3 \pm 7.8$ beats $/ \mathrm{min}$ $(23.85 \%)$ in heart rate $(P<0.001)$ at 6 hours after dosing (Figure 2$)$.

\section{Pharmacokinetic analysis}

Following oral administration of $2 \mathrm{mg}$ terazosin, the log plasma concentration-time profile of terazosin in 21 healthy subjects was shown in Figure 3, with the pharmacokinetic parameters summarized in Table 1. The pharmacokinetic parameters are consistent with the previous reports, except V/F $[9,13]$. The median time to reach $\mathrm{C}_{\max }$ was $0.89 \pm 0.62$ hours. The plasma concentration of terazosin decreased after $\mathrm{t}_{\max }$ till 48 hours, when the plasma concentration of terazosin was measureable as $1.03 \pm 0.38 \mathrm{ng} / \mathrm{mL}$. The half-life of terazosin

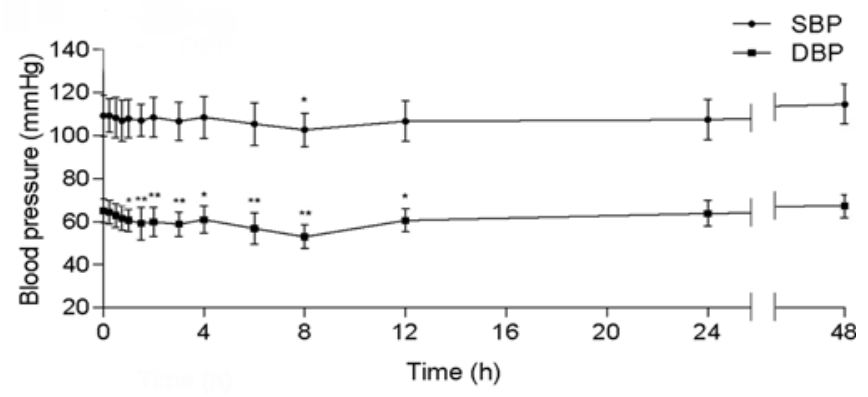

Figure 1: Effect of terazosin on blood pressure following the administration of $2 \mathrm{mg}$ terazosin in 21 healthy subjects $\left(n=21\right.$, mean \pm SD). ${ }^{*} P<0.05,{ }^{* *} P<0.01$ compared with pre-dosing.

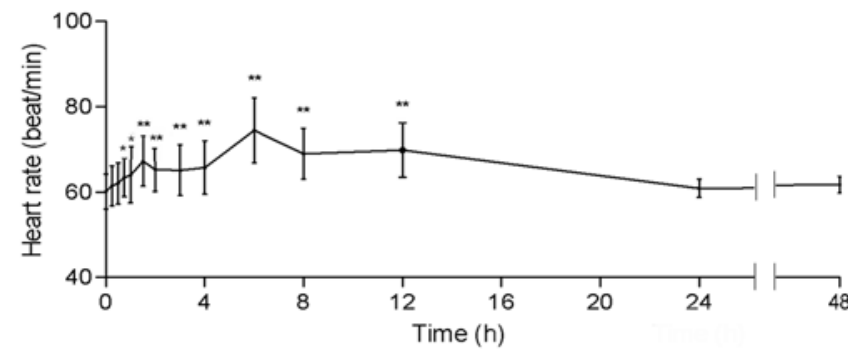

Figure 2: Effect of terazosin on heart rate following the administration of $2 \mathrm{mg}$ terazosin in 21 healthy subjects $\left(n=21\right.$, mean \pm SD). ${ }^{*} P<0.05$, ${ }^{* *} P<0.01 \mathrm{com}-$ pared with pre-dosing.

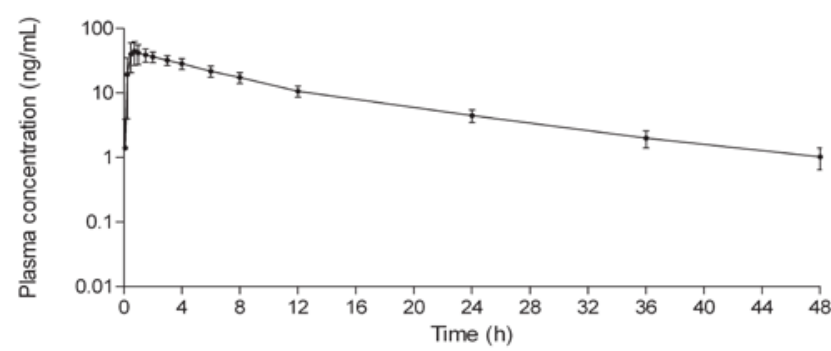

Figure 3: Log plasma concentration-time profile of terazosin following the administration of $2 \mathrm{mg}$ terazosin in 21 healthy subjects ( $n=21$, mean $\pm \mathrm{SD})$.

\begin{tabular}{|c|c|}
\hline Parameter & Value \\
\hline $\mathrm{C}_{\text {max }}(\mathrm{ng} / \mathrm{mL})$ & $51.39 \pm 13.29$ \\
\hline $\mathrm{T}_{\max }(\mathrm{h})$ & $0.89 \pm 0.62$ \\
\hline $\mathrm{t}_{1 / 2}(\mathrm{~h})$ & $10.21 \pm 1.41$ \\
\hline $\mathrm{Kel}(1 / \mathrm{h})$ & $0.069 \pm 0.009$ \\
\hline $\mathrm{CL} / \mathrm{F}(\mathrm{L} / \mathrm{h})$ & $4.65 \pm 0.81$ \\
\hline $\mathrm{V} / \mathrm{F}(\mathrm{L})$ & $68.22 \pm 13.57$ \\
\hline $\mathrm{AUC}_{0-\mathrm{t}}(\mathrm{ng}-\mathrm{h} / \mathrm{mL})$ & $428.92 \pm 79.39$ \\
\hline $\mathrm{AUC}_{0-\infty}(\mathrm{ng}-\mathrm{h} / \mathrm{mL})$ & $442.69 \pm 83.55$ \\
\hline
\end{tabular}

Table 1: Mean pharmacokinetic parameters of terazosin following the administration of $2 \mathrm{mg}$ terazosin in 21 healthy subjects $(n=21$, mean \pm SD).

was $10.21 \pm 1.41$ hours. Kel and CL/F of terazosin were $0.069 \pm 0.009$ and $4.65 \pm 0.81 \mathrm{~L} / \mathrm{h}$, respectively. $\mathrm{V} / \mathrm{F}$ of terazosin was $68.22 \pm 13.57$ L, different from the previous study $[9,13]$. Theoretically, these pharmacokinetic results indicated that terazosin could have a relatively rapid onset of action and sufficiently long duration of action.

\section{Safety evaluation}

Terazosin was well tolerated in 22 healthy subjects. No serious adverse events occurred during the study. Mild nasal obstruction in two subjects $(9.1 \%)$ and mild palpitation in one subject (4.6\%) were reported, which was considered probably related to terazosin. In clinical laboratory test before discharge, mild hyperuricaemia in one subject (4.6\%) was not considered as related to terazosin. Mild leucopenia in one subject (4.6\%) was possibly related to terazosin. All adverse events were followed up until the results became normal. 


\section{Discussion}

Following oral administration of $2 \mathrm{mg}$ terazosin, plasma concentration of terazosin rapidly reached to peak concentration, and then decreased slowly. The prolonged half-life (10.21 \pm 1.41 hours) made it lower the blood pressure for longer time than other short-term antihypertensive drugs, allowing once-daily dosing. In summary, the pharmacokinetic properties of terazosin from healthy Chinese male subjects were consistent with the results from South Korea and healthy Spanish subjects $[13,14]$.

Previous studies indicated that the daily dose of terazosin, which produced $50 \%$ of the maximum response $\left(\mathrm{ED}_{50}\right)$, was $3.0 \mathrm{mg}$ for $\mathrm{SBP}$ and $1.5 \mathrm{mg}$ for DBP in hypertensive patients [1]. Blood pressure, as the pharmacodynamic evaluation of antihypertensive drugs, has individual variation and normal fluctuation throughout the day [1]. In the present study, single-dose of $2 \mathrm{mg}$ terazosin produced the considerable blood pressure reduction, especially DBP in healthy Chinese subjects. SBP showed a significant reduction $(P<0.05)$ only at 8 hours, whereas DBP had a significant reduction $(P<0.05)$ at the time intervals of $1-12$ hours after dosing. The degree of decrease in DBP was also stronger than SBP, with $8.5 \pm 5.3 \mathrm{mmHg}(7.78 \%)$ and $12.9 \pm 6.0 \mathrm{mmHg}(19.82 \%)$ at 8 hours after dosing, respectively. According to the normal fluctuation rule, blood pressure was close to the second peak at $4 \mathrm{pm}$ ( 8 hours after dosing). It suggested that terazosin exerted its significantly antihypertensive effect in healthy Chinese subjects.

Campanero et al. [13] had developed the pharmacokinetic/ pharmacodynamic modeling of terazosin in healthy Spanish subjects. The present study investigated the correlation of pharmacokinetic and pharmacodynamic response of terazosin in healthy Chinese subjects. Obtaining the multiple data from every subject allowed for estimating the pharmacodynamic property of terazosin. Thus, we measured the blood pressure for fourteen times, except 0.1 and 36 hours after dosing. The time points were almost consistent with sampling points, in order to accurately evaluate the correlation between the antihypertensive effect and plasma concentration of terazosin. The findings showed the significant hysteresis phenomenon, with the lagging antihypertensive effect behind plasma concentration of terazosin. When plasma concentration of terazosin rapidly reached to peak concentration after dosing, blood pressure showed the slight reduction. When plasma concentration of terazosin decreased nearly $50 \%$, the drug exerted the maximum antihypertensive effect. The maximum blood pressure reduction occurred at 7 hours later than $T_{\text {max }}(0.89 \pm 0.62 \mathrm{~h})$. However, the lagging time was 1.2-1.8 hours later than $\mathrm{T}_{\max }(1.27 \pm 0.11$ h) in healthy Spanish subjects [13]. Maybe terazosin had the different antihypertensive effect in the different races, as resulted in the different lagging time. The racial difference was also indicated in the discrepancy of drug response and clearance $[15,16]$.

In addition, a decrease in arterial blood pressure triggers a reflexive increase in heart rate through the increased sympathetic activity. 2 $\mathrm{mg}$ terazosin caused the increase in heart rate from 0.25 hours till 24 hours after dosing in healthy Chinese subjects. The maximum increase of $14.3 \pm 7.8$ beats $/ \mathrm{min}(23.85 \%)$ occurred at 6 hours, close to the time of the maximum antihypertensive effect. In healthy Spanish subjects, the most significant increase in heart rate occurred after 3 hours by $6.1 \pm 2.3$ beats/min without significant change in 3 hours after dosing [13]. Because both the healthy Chinese and Spanish subjects had the similar $\mathrm{C}_{\max }$ at about $1 \mathrm{~h}$, the Chinese subjects had more significant lagging in antihypertensive effect, as well as increase in heart rate than Spanish subjects.
The present findings were valuable for the clinical doctors to recipe this drug for the hypertensive patients to take it in China. It also supplied the safety data for pharmacokinetic or bioequivalence studies in healthy volunteers. Close attention should be paid to blood pressure reduction and heart rate increase during 6-8 hours after oral administration of terazosin for fear of the occurrence of adverse reactions. In the present study, we took measures to protect the safety of the subjects, including prolonging the time of lying in bed and remaining in the clinical research unit, enhancing the clinical monitoring and nursing. During the study period, no serious adverse events occurred and terazosin was well tolerated in healthy Chinese male volunteers.

In conclusion, the present results suggested a significant hysteresis phenomenon of antihypertensive effect behind the plasma concentration of terazosin in healthy Chinese subjects. It was different from other racial subjects. Further studies will be conducted to investigate the mechanisms that terazosin, as $\alpha_{1}$-adrenergic receptor, has significant lagging in the antihypertensive effect in healthy Chinese subjects.

\section{Acknowledgments}

This work was supported by Shijiazhuang Yiling pharmaceutical Co. Ltd. (Shijiazhuang, China)

\section{References}

1. Achari R, Hosmane B, Bonacci E, O'Dea R (2000) The relationship between terazosin dose and blood pressure response in hypertensive patients. J Clin Pharmacol 40: 1166-1172.

2. Tsai YS, Lan SK, Ou JH, Tzai TS (2007) Effects of branded versus generic terazosin hydrochloride in adults with benign prostatic hyperplasia: a randomized, open-label, crossover study in Taiwan. Clin Ther 29: 670-682.

3. Alan C, Kirilmaz B, Kocoglu H, Ersay AR, Ertung Y, et al. (2011) Comparison of effects of alpha receptor blockers on endothelial functions and coagulation parameters in patients with benign prostatic hyperplasia. Urology 77: 14391443

4. Liu H, Liu P, Mao G, Chen G, Wang B, et al. (2009) Efficacy of combined amlodipine/terazosin therapy in male hypertensive patients with lower urinary tract symptoms: a randomized, double-blind clinical trial. Urology 74: 130-136.

5. Low BY, Liong ML, Yuen KH, Chee C, Leong WS, et al. (2008) Terazosin therapy for patients with female lower urinary tract symptoms: a randomized double-blind, placebo controlled trial. J Urol 179: 1461-1469.

6. Ligueros M, Unwin R, Wilkins MR, Humphreys J, Coles SJ, et al .(1992) A comparison of the effects of the selective peripheral alpha 1-blocker terazosin with the selective beta 1-blocker atenolol on blood pressure, exercise performance and the lipid profile in mild-to-moderate essential hypertension. Clin Auton Res 2: 373-381.

7. Kondo K, Ojashi K, Ebihara A (1982) The pharmacokinetics and pharmacological effects of terazosin, a new a-blocking agent, in normotensive volunteers. Jap Clin Pharmacol Therap 13: 137-138.

8. Titmarsh S, Monk JP (1987) Terazosin. A review of its pharmacodynamic and pharmacokinetic properties, and therapeutic efficacy in essential hypertension. Drugs 33: 461-477.

9. Sonders RC (1986) Pharmacokinetics of terazosin. Am J Med 80: 20-24

10. Jungers P, Ganeval D, Pertuiset N, Chauveau P (1986) Influence of renal insufficiency on the pharmacokinetics and pharmacodynamics of terazosin. Am J Med 80: 94-99.

11. McNeil JJ, Drummer OH, Conway EL, Workman BS, Louis WJ (1987) Effect of age on pharmacokinetics of and blood pressure responses to prazosin and terazosin. J Cardiovasc Pharmacol 10: 168-175.

12. Cheah PY, Yuen KH, Liong ML (2000) Improved high-performance liquid chromatographic analysis of terazosin in human plasma. J Chromatogr B Biomed Sci Appl 745: 439-443. 
Citation: Qiang G, Yang M, Zhang Y, Liu M, Zhang D, et al. (2011) Pharmacokinetic and Pharmacodynamic Study of Terazosin in Healthy Chinese Volunteers: Significant Hysteresis Phenomenon. J Bioequiv Availab 3: 228-232. doi:10.4172/jbb.1000091

13. Campanero MA, Sadaba B, Munoz-Juarez MJ, Quetglas EG, Azanza JR (2008) Pharmacokinetic and pharmacodynamic modelling of arterial haemodynamic effects of terazosin in healthy volunteers. Clin Drug Investig 28: 139-147.

14. Kang BC, Yang CQ, Rhee JE, Suh OK, Shin WG (2001) High-performance liquid chromatographic analysis and pharmacokinetics of terazosin in healthy volunteers. Res Commun Mol Pathol Pharmacol 110: 371-377.
15. Small DS, Payne CD, Kothare P, Yuen E, Natanegara F, et al. (2010) Pharmacodynamics and pharmacokinetics of single doses of prasugrel $30 \mathrm{mg}$ and clopidogrel $300 \mathrm{mg}$ in healthy Chinese and white volunteers: an open-labe trial. Clin Ther 32: 365-379.

16. Bigos KL, Bies RR, Pollock BG, Lowy JJ, Zhang F, et al. (2011) Genetic variation in CYP3A43 explains racial difference in olanzapine clearance. Mol Psychiatry 16: 620-625. 